\title{
A-PROPERNESS AND FIXED POINT THEOREMS FOR DISSIPATIVE TYPE MAPS
}

\author{
K. Q. LAN AND J. R. L. WEBB
}

Received 26 April 1999

We obtain new $A$-properness results for demicontinuous, dissipative type mappings defined only on closed convex subsets of a Banach space $X$ with uniformly convex dual and which satisfy a property called weakly inward. The method relies on a new property of the duality mapping in such spaces. New fixed point results are obtained by utilising a theory of fixed point index.

\section{Introduction}

The A-proper class arises naturally when one considers the approximation solvability of nonlinear equations, that is, obtaining solutions of infinite-dimensional problems as limits of solutions of related finite-dimensional problems. The class was first introduced by Petryshyn, who made many important contributions to the theory, see, for example, $[17,18]$ for a good account of this. The $A$-proper class is large, it allows various classes of nonlinear mappings to be treated in a unified way and has found wide applications.

In a recent paper, the present authors have developed a new theory of fixed point index for weakly inward $A$-proper maps. (Precise definitions of concepts mentioned in the introduction are given later.) This allows fixed point theorems and mapping theorems to be proved for $A$-proper maps whose domain is a subset of a Banach space $X$. Many of the standard examples of $A$-proper maps have domains the whole space, for example maps of the form $I-f$ where $f$ is $k$-ball contractive [20] or accretive type maps [21]. If $D$ is a closed subset of a Banach space $X$ and $f: D \rightarrow X$, it is harder to establish $A$-properness. For example, when $f: D \rightarrow X$ is a strict contraction, that is, $\|f(x)-f(y)\| \leq k\|x-y\|(k<1)$ for $x, y \in D$, an open problem posed by Petryshyn is whether $I-f$ is $A$-proper. This is known to hold if $D=X$ or with some extra conditions on $D$ and $f$ in a reflexive space $X$. Recently the authors have shown that $I-f$ is $A$-proper at 0 when $f: D \rightarrow D$ and $D$ is a closed set in an arbitrary Banach space [12].

Copyright $\odot 1999$ Hindawi Publishing Corporation

Abstract and Applied Analysis 4:2 (1999) 83-100

1991 Mathematics Subject Classification: 47H09, 47H06

URL: http://aaa.hindawi.com/volume-4/S108533759900010X.html 
In the present paper, we consider maps of accretive and dissipative type, $(A$ is accretive if and only if $-A$ is dissipative) defined on a closed convex subset of $X$. We suppose $X$ is a $\pi_{1}$-Banach space, that is, has a suitable projection scheme, and (usually) that $X^{*}$ is uniformly convex (to ensure that the duality map has good properties). We establish new results on $A$-properness of these maps when they are weakly inward. This gives the first result for demicontinuous maps of this type when the domain is not the whole space and the space is not a Hilbert space.

The weakly inward condition arises naturally for accretive operators. If $D$ is a closed convex set and $A: D \rightarrow X$ is a continuous accretive map, a key assumption in establishing global solutions of the evolution equation

$$
\frac{d u}{d t}+A u=f, \quad u(0)=x \in D
$$

is the following tangency condition of flow invariance:

$$
\lim _{h \rightarrow 0^{+}} \frac{\operatorname{dist}(x-h A x, D)}{h}=0 \quad \text { for } x \in D .
$$

When $D$ is a closed convex set it is known that this is equivalent to saying that $I-A$ is weakly inward. Equation (1.2) is more stringent than our definition of $I-A$ weakly inward (given below) when applied to a closed set $D$. However, we only consider the weakly inward property relative to closed convex sets in this paper.

Petryshyn considered the $A$-properness of a general class of maps called $K$-monotone [16]. When applied to a strongly accretive map it gave an $A$-properness result when $X$ is locally uniformly convex, and the duality map is weakly continuous, a condition that is known to be rather restrictive outside of Hilbert space.

When $D=X$, Webb [21] proved an $A$-proper result for demicontinuous strongly accretive maps in spaces with uniformly convex duals, by showing that the duality map satisfied a condition that can replace weak continuity.

In Hilbert spaces, Benavides [2] obtained a definitive result. He showed that a bounded, demicontinuous, and strongly accretive map $A$ defined on a closed subset $D$ is $A$-proper without any further assumptions on the map or on the domain $D$. However, his proof makes essential use of the Hilbert space structure.

In this paper, we obtain $A$-properness for weakly inward maps, we call $\psi$-dissipative that are either continuous or bounded and demicontinuous. This includes contractive type maps. A precursor of our results is the result of [22] that, if $f$ is a generalized contraction defined only on a closed convex subset of a reflexive $\pi_{1}$-space and $f$ is weakly inward, then $I-f$ is $A$-proper at 0 . This gave a partial answer to the open question mentioned earlier.

The main tool in our work is a new property of the normalized duality map $J$ which is proved by a combination of the methods of [21, 22].

The newly developed theory of fixed point index mentioned above is now applicable. Difficulties can arise when trying to use the homotopy property of index to treat unbounded maps. We avoid these here by only using the index theory in finite dimensions, carrying out the homotopy at the finite-dimensional level. This is a known idea in the theory of $A$-proper maps, for example, see [18, Section 4.4]. 
As applications of our new results on $A$-properness, we derive some new fixed point and mapping theorems which give new versions of some results of Martin [15] under weaker continuity hypotheses and obtain approximation solvability results, that is, our solutions are obtained as limits of solutions of related finite-dimensional problems. Our results also improve a result of Deimling [5]. They provide extensions and improvements of some of the results of Lafferriere and Petryshyn [10] where more stringent restrictions are imposed. Their maps are defined on a cone, and they require that the map satisfies a strong range condition (see $\left(\mathrm{H}_{4}\right)$ in Proposition 1.1 of [10]).

For continuous maps defined only on a subset of $X$, it is possible to prove $A$ properness for dissipative type maps by making use of existence theorems proved by other methods, see, for example, [22, 23]. However, A-proper methodology aims to deduce such existence theorems from simpler finite-dimensional results and we follow that aim here.

We give an application of our new results to an integral equation, versions of which have been studied, for example, in $[10,11]$.

\section{Prerequisites and remarks on weakly inward maps}

A Banach space is denoted by $X$ with dual space $X^{*}$. The class of $A$-proper maps is defined in terms of certain approximation schemes. Suppose that there exist a sequence of finite-dimensional subspaces $X_{n} \subset X$ and a sequence of continuous linear projections $P_{n}: X \rightarrow X_{n}$ such that $P_{n} x \rightarrow x$ for each $x \in X . \Gamma=\left\{X_{n}, P_{n}\right\}$ is called a projection scheme for $X$. Obviously, such a space is separable and we have $\sup _{n \in N}\left\|P_{n}\right\|=c<\infty$ by the uniform boundedness principle. $X$ is said to be a $\pi_{c}$-space and is called a $\pi_{1}$ space when $\left\|P_{n}\right\|=1$ for every $n$. It is known that every Banach space with a monotone Schauder basis is a $\pi_{1}$-space, which includes the commonly occurring spaces, for example, $c_{0}, l^{p}, 1 \leq p<\infty, C[0,1]$ and $L^{p}[0,1]$ for $1 \leq p<\infty$ (cf. [14]).

When discussing the $A$-properness of maps in this paper we always assume that $X$ is a $\pi_{c}$-space, usually a $\pi_{1}$-space.

Definition 2.1. A map $A: D \subset X \rightarrow X$ is said to be approximation-proper ( $A$-proper, for short) at $q \in X$ if $\left.A_{n} \equiv P_{n} A\right|_{D \cap X_{n}}$ is continuous for each $n \in \mathbb{N}$ and whenever $\left\{x_{n_{k}}: x_{n_{k}} \in D \cap X_{n_{k}}\right\}$ is bounded and $A_{n_{k}} x_{n_{k}} \rightarrow q$, then $\left\{x_{n_{k}}\right\}$ has a subsequence which converges to some $x \in D$ with $A x=q$. $A$ is said to be $A$-proper on a set $K$ if it is $A$-proper at every point $q \in K$.

A map $A: D \rightarrow X$ is said to be demicontinuous if $x_{n} \rightarrow x \in D$ implies that $A x_{n} \rightarrow A x$. Here, and later, $\rightarrow$ denotes weak convergence. We note that $A: D \rightarrow X$ demicontinuous implies that $A_{n}: D_{n}:=D \cap X_{n} \rightarrow X_{n}$ is continuous.

The normalized (multivalued) duality map $J: X \rightarrow 2^{X^{*}}$ is defined by

$$
J(x):=\left\{f \in X^{*}:\|f\|=\|x\| \text { and }(x, f)=\|x\|^{2}\right\} .
$$

(Here and henceforth $(x, f)$ denotes $f(x)$.) When $X^{*}$ is strictly convex, $J(x)$ is a 
singleton and $J$ is a single-valued map. In this paper, we assume either that $X^{*}$ is uniformly convex or that $X$ is reflexive and has a uniformly Gateaux-differentiable norm (we write $X$ is (UG)); $X^{*}$ uniformly convex implies $X$ is reflexive and (UG) which implies $X^{*}$ is strictly convex. The reasons for making these assumptions are the following known properties of $J$ (when $X^{*}$ is strictly convex).

$\left(\mathrm{J}_{1}\right)(y-x, J x) \leq\left(\|y\|^{2}-\|x\|^{2}\right) / 2$, for all $x, y \in X$.

In fact this follows immediately from $(y, J x) \leq\|y\|\|x\| \leq\left(\|y\|^{2}+\|x\|^{2}\right) / 2$.

$\left(\mathrm{J}_{2}\right)$ If $X$ is a $\pi_{1}$-space, then $P_{n}^{*} J(x)=J(x)$ for each $x \in X_{n}$ (see [4], or [17, Lemma 2.5, page 32]).

$\left(\mathrm{J}_{3}\right)$ If $X$ is reflexive, $J$ is surjective, that is, $J(X)=X^{*}$ (cf. [6, Theorem 12.3] or [15, Proposition I.6.2]).

$\left(\mathrm{J}_{4}\right)$ If $X^{*}$ is uniformly convex, $J$ is continuous, uniformly on bounded subsets of $X$ (a result of Kato, cf. [6, Proposition 12.3]).

$\left(\mathrm{J}_{5}\right)$ If $X$ is reflexive and (UG), then $J$ is demicontinuous, uniformly on bounded subsets of $X$.

We are discussing weakly inward maps. These have been studied by a number of authors including Halpern and Bergman [7], and are defined as follows. Let $K$ be a closed convex set in $X$. For $x \in K$ let

$$
I_{K}(x)=\{x+c(y-x): y \in K \text { and } c \geq 1\} .
$$

$I_{K}(x)$ is called the inward set of $x$ relative to $K$. By convexity of $K, I_{K}(x)$ is also equal to $\{x+c(y-x): y \in K$ and $c \geq 0\}$. Its closure is written $\bar{I}_{K}(x)$. Recall that a closed convex set $K$ is called a wedge if $\lambda x \in K$ for all $x \in K$ and $\lambda \geq 0$; a wedge $K$ is called a cone if also $K \cap(-K)=\{0\} . I_{K}(x)$ is a convex set containing $K$ and $\bar{I}_{K}(x)$ is a wedge if $K$ is a wedge.

Definition 2.2. A map $A: D \subseteq K \rightarrow X$ is said to be weakly inward (relative to $K$ ) if $A(x) \in \bar{I}_{K}(x)$ for every $x \in D$.

Note that, when $K$ is convex, $I_{K}(x)$ is the union of all rays starting at $x$ and passing through some other point of $K$. Hence $I_{K}(x)=X$ if $x$ is an interior point of $K$ so saying that $A$ is weakly inward corresponds to a boundary condition. Other equivalent definitions of weakly inward are possible, for example, a definition may be given in terms of support functionals, which is sometimes easier to check. For details we refer to [6, Sections 18.3 and 20.4]. We utilize the following in our application in Section 6.

Lemma 2.3. $A: K \rightarrow X$ is weakly inward on a cone $K$ if

$$
x \in \partial K, x^{*} \in K^{*} \text {, and }\left(x, x^{*}\right)=0 \text { together imply }\left(A x, x^{*}\right) \geq 0 \text {. }
$$

Here $K^{*}=\left\{x^{*} \in X^{*}:\left(x, x^{*}\right) \geq 0\right.$ on $\left.K\right\}$, is the so-called dual cone (but actually it is a wedge).

We first give some simple observations concerning weakly inward maps.

Lemma 2.4 (see [13]). Let $K$ be a closed convex set in a Banach space $X$. If $w \in \bar{I}_{K}(x)$, then $(1-a) x+a w \in \bar{I}_{K}(x)$ for every $a>0$. 
Proof. Let $w=\lim w_{n}$ where $w_{n}=\left(1-c_{n}\right) x+c_{n} y_{n}, y_{n} \in K, c_{n} \geq 0$. Then

$$
(1-a) x+a w_{n}=\left(1-a c_{n}\right) x+a c_{n} y_{n} \in I_{K}(x)
$$

so that $(1-a) x+a w \in \bar{I}_{K}(x)$.

Proposition 2.5. Let $K$ be a closed convex set in $X$. The following statements are equivalent:

(i) $w \in \bar{I}_{K}(x)$.

(ii) There exists $b>0$ such that $(1-b) x+b w \in \bar{I}_{K}(x)$.

(iii) $(1-b) x+b w \in \bar{I}_{K}(x)$ for all $b>0$.

Proof. By Lemma 2.4, (i) $\Rightarrow$ (iii). Obviously (iii) $\Rightarrow$ (ii). If (ii) holds, then by Lemma 2.4, $(1-a) x+a[(1-b) x+b w] \in \bar{I}_{K}(x)$ for every $a>0$. Take $a=1 / b$ to g et $w \in \bar{I}_{K}(x)$.

Proposition 2.6. Let $K$ be a closed convex set in $X . I+f$ is weakly inward (relative to $K$ ) if and only if $I+$ af is weakly inward for all $a>0$.

Proof. $x+a f(x)=(1-a) x+a(I+f)(x)$; apply Proposition 2.5.

Sums of weakly inward maps need not be weakly inward. The correct result is the following.

Proposition 2.7. Let $K$ be a closed convex set in a Banach space $X$. If $I+f$ and $I+g$ are weakly inward, then so is $I+a f+b g$ for every $a, b \geq 0$.

Proof. Since $I+f$ is weakly inward we have, by Proposition 2.6, $I+2 a f$ is weakly inward and similarly so is $I+2 b g$. By convexity of $\bar{I}_{K}(x)$, this yields $[I+2 a f] / 2+$ $[I+2 b g] / 2$ is weakly inward.

Proposition 2.8. If $0 \in K$, then $I+a_{0} f$ weakly inward for some $a_{0}>0$ implies that $(1-b) I+a f$ is weakly inward for all $a \geq 0, b \geq 0$.

Proof. Let $g: K \rightarrow X$ be defined by $g(x)=-x$. Then $I+g$ is weakly inward since $0 \in K$. By Proposition 2.7, we have $I+a f+b g=a f+(1-b) I$ is weakly inward for all $a, b \geq 0$.

When $K$ is a wedge, $\bar{I}_{K}(x)$ is also a wedge and this gives $f$ weakly inward relative to a wedge $K$ if and only if $I+f$ is weakly inward if and only if $(1-b) I+a f$ is weakly inward for all $a, b \geq 0$.

This result is false for a convex set $K$, in general, even if $K$ contains 0 . For example if $K=\bar{B}(0,1)$, the closed unit ball of $X$, and $f(x)=x$, then $f$ is weakly inward but $I+f$ is not. (It fails even in $\mathbb{R}$.)

Remark 2.9. These results may be deduced from the support functional definition, and when the maps are continuous from [15, Proposition 2.4, page 215]. 


\section{A new property of duality maps}

Our new property of $J$ reads as follows.

Proposition 3.1. Let $X$ be a reflexive (UG) Banach space and let $K$ be a closed convex subset of $X$. Let $\left\{x_{n}\right\} \subset X$ be a bounded sequence. Then there exists $v \in K$ such that

$$
\liminf _{n \rightarrow \infty}\left(y-v, J\left(x_{n}-v\right)\right) \leq 0, \quad \text { for every } y \in \bar{I}_{K}(v) .
$$

Proof. For each $z \in X$, let

$$
\phi(z)=\limsup _{n \rightarrow \infty}\left\|x_{n}-z\right\|
$$

Then $\phi$ is a continuous convex function and $\phi(z) \rightarrow \infty$ as $\|z\| \rightarrow \infty$. Therefore $\phi$ attains its infimum over the closed convex set $K$, that is, there is $v \in K$ such that $\phi(v)=\inf \{\phi(z): z \in K\}$. We prove that

$$
\phi(v)=\inf \left\{\phi(z): z \in \bar{I}_{K}(v)\right\} .
$$

Let $r=\inf \left\{\phi(z): z \in \bar{I}_{K}(v)\right\}$ and suppose that $r<\phi(v)$. Let $\varepsilon>0$ be such that $r+\varepsilon<\phi(v)$. Since $\phi$ is continuous, there exists $z \in I_{K}(v)$ such that $\phi(z) \leq r+\varepsilon$. Thus $z=v+c(w-v)$ for some $w \in K$ and $c \geq 1$. By convexity of $\phi$, we have

$$
\phi(v) \leq \phi(w) \leq\left(\frac{1}{c}\right) \phi(z)+\left(1-\left(\frac{1}{c}\right)\right) \phi(v) .
$$

Hence $\phi(v) \leq \phi(z) \leq r+\varepsilon$, a contradiction. This shows that $r=\phi(v)$.

For arbitrary $y \in \bar{I}_{K}(v)$ and $t>0$ let $z_{t}=(1-t) v+t y$. Then $z_{t} \in \bar{I}_{K}(v)$. By property $\left(\mathrm{J}_{1}\right)$, we have

$$
2\left(z_{t}-v, J\left(x_{n}-z_{t}\right)\right) \leq\left\|x_{n}-v\right\|^{2}-\left\|x_{n}-z_{t}\right\|^{2} .
$$

This implies

$$
2 t \liminf _{n \rightarrow \infty}\left(y-v, J\left(x_{n}-z_{t}\right)\right) \leq \phi^{2}(v)-\phi^{2}\left(z_{t}\right) \leq 0 .
$$

Cancelling $t>0$, letting $t \rightarrow 0+$ and using $\left(\mathrm{J}_{5}\right)$, we obtain

$$
\liminf _{n \rightarrow \infty}\left(y-v, J\left(x_{n}-v\right)\right) \leq 0 .
$$

Remark 3.2. This result is related to a result of Baillon, see [6, Proposition 14.1(c)]. Our new idea is to use the idea from [22] to show that (3.1) holds for $y \in \bar{I}_{K}(v)$, not just for $y \in K$.

When $X$ is separable, by a diagonalization argument, given a bounded sequence $\left\{x_{n}\right\}$ there is a subsequence $\left\{x_{k}\right\}$ such that $\lim \left\|x_{k}-z\right\|$ exists for every $z \in X,[19]$. In that case we have the following result. 
Proposition 3.3. Let $X$ be a separable, reflexive (UG) Banach space and let $K$ be a closed convex subset of $X$. For a bounded sequence $\left\{x_{n}\right\} \subset X$ there is a subsequence $\left\{x_{n_{k}}\right\}$ and a point $v \in K$ such that

$$
\limsup _{k \rightarrow \infty}\left(y-v, J\left(x_{n_{k}}-v\right)\right) \leq 0, \quad \text { for every } y \in \bar{I}_{K}(v) .
$$

Proof. We follow the proof of Proposition 3.1 save that we take

$$
\phi(z):=\lim _{k \rightarrow \infty}\left\|x_{n_{k}}-z\right\| .
$$

If $K=X$, then $\bar{I}_{K}(v)=X$, hence $J\left(x_{n_{k}}-v\right) \rightarrow 0$. Therefore, Proposition 3.3 generalizes the proposition in [21].

\section{A-properness of accretive mappings}

We now suppose that $X$ is a $\pi_{1}$-space. A map $A: D \subseteq X \rightarrow X$ is said to be accretive if for each $x, y \in D$ there exists $f \in J(x-y)$ such that $(A(x)-A(y), f) \geq 0$. This is equivalent to $\|x+r A(x)-y-r A(y)\| \geq\|x-y\|$ for every $r>0$. $A$ is said to be strongly accretive if there is a constant $c>0$ such that $(A(x)-A(y), f) \geq c\|x-y\|^{2}$.

For $D=X$, Webb [21] was able to prove an $A$-properness result for strongly accretive maps in spaces with uniformly convex duals, by utilising the remark following Proposition 3.3 to replace weak continuity of the duality map. In the present paper, we use Proposition 3.1 to extend this result to allow a more general accretive map $A$ and the domain of $A$ to be an arbitrary closed, convex set $K$ when $I-A$ is weakly inward on $K$.

In Hilbert spaces, the definitive result has been given by Benavides [2]: a bounded, demicontinuous, and strongly accretive map $A$ defined on a closed subset $D$ is $A$-proper. No further assumption on the map or on the domain $D$ is needed. However, the proof makes essential use of the Hilbert space structure.

We prove a result for a new class of maps we call $s$-accretive.

Definition 4.1. A map $A: D \rightarrow X$ is said to be $s$-accretive if whenever $\left\{x_{n}\right\} \subset D$ is a bounded sequence and

$$
\liminf _{n \rightarrow \infty}\left(A x_{n}-A y, J\left(x_{n}-y\right)\right) \leq 0 \quad \text { for some } y \in D
$$

then there is a subsequence $x_{n_{k}} \rightarrow y$.

This has some similarities with the class $S_{+}$, see, for example, [17], but it is important for us that it is not assumed that $x_{n} \rightarrow y$. We first show that, this class includes many other well-known classes.

Example 4.2. Suppose that $A: D \rightarrow X$ is $\alpha$-accretive, that is,

$$
(A x-A y, J(x-y)) \geq \alpha(y,\|x-y\|), \quad x, y \in D,
$$


where $\alpha: D \times[0, \infty) \rightarrow \mathbb{R}$ satisfies $\alpha(y, r)>0$ for $r>0$, and $\alpha(y, \cdot)$ is lower semicontinuous (lsc), that is, $r_{n} \rightarrow r$ implies $\alpha(y, r) \leq \liminf \alpha\left(y, r_{n}\right)$. Then $A$ is $s$-accretive.

In fact, suppose that $y \in D$ and $\liminf _{n \rightarrow \infty}\left(A x_{n}-A y, J\left(x_{n}-y\right)\right) \leq 0$. Then there is a subsequence, denoted $\left\{x_{k}\right\}$, such that

$$
\lim _{k \rightarrow \infty}\left(A x_{k}-A y, J\left(x_{k}-y\right)\right)=\liminf _{n \rightarrow \infty}\left(A x_{n}-A y, J\left(x_{n}-y\right)\right)
$$

and $\phi(y):=\lim \sup \left\|x_{n}-y\right\|=\lim \left\|x_{k}-y\right\|$. Then we have

$$
\liminf _{k \rightarrow \infty} \alpha\left(y,\left\|x_{k}-y\right\|\right) \leq \lim _{k \rightarrow \infty}\left(A x_{k}-A y, J\left(x_{k}-y\right)\right) \leq 0 .
$$

Using the lsc we obtain $\alpha(y, \phi(y)) \leq 0$ so that $\phi(y)=0$ and thus $x_{k} \rightarrow y$.

Obviously the class of $\alpha$-accretive maps includes strongly accretive maps.

Definition 4.3. A map $T: D \rightarrow X$ is said to be $\psi$-dissipative if

$$
(T x-T y, J(x-y)) \leq \psi(y,\|x-y\|) \quad \text { for } x, y \in D
$$

where $\psi: D \times[0, \infty) \rightarrow \mathbb{R}$ satisfies $\psi(y, r)<r^{2}$ for each $y \in D$ and $r>0$ and $\psi(y, \cdot)$ is upper semicontinuous (usc), that is, if $r_{n} \rightarrow r$, then $\psi(y, r) \geq \lim \sup \psi\left(y, r_{n}\right)$.

Clearly we have the following result.

Proposition 4.4. A map $T: D \rightarrow X$ is $\psi$-dissipative if and only if $I-T$ is $\alpha$-accretive with $\alpha(y, r)=r^{2}-\psi(y, r)$.

This shows that $I-T$ is $s$-accretive when $T$ is $\psi$-dissipative. This new class of $\psi$-dissipative maps includes contractive maps. In fact we have the following result.

Example 4.5. Let $T: D \rightarrow X$ be a generalized contraction [8], that is, for each $y \in D$ there is $k(y)<1$ such that $\|T(x)-T(y)\| \leq k(y)\|x-y\|$ for all $x \in D$. Then $I-T$ is $s$-accretive.

Indeed, $T$ is $\psi$-dissipative with $\psi(y, r)=k(y) r^{2}$. Now we give our first $A$-properness result. This is a new result because we consider $s$-accretive maps but it is new even for strongly accretive operators. Recall that a map $A: D \rightarrow X$ is said to be bounded if it maps bounded sets into bounded sets.

Theorem 4.6. Let $X$ be a $\pi_{1}$-space with $X^{*}$ uniformly convex and let $K$ be a closed convex set. Let $A: K \rightarrow X$ be a bounded, demicontinuous, $s$-accretive map and suppose that $I-A$ is weakly inward relative to $K$. Then $A$ is A-proper at 0 . 
Proof. Let $x_{n} \in K_{n}$ be bounded and such that $P_{n} A x_{n} \rightarrow 0$. By Proposition 3.1 there exists $v \in K$ such that

$$
\liminf _{n \rightarrow \infty}\left(y-v, J\left(x_{n}-v\right)\right) \leq 0, \quad \text { for every } y \in \bar{I}_{K}(v) .
$$

As $v-A v \in \bar{I}_{K}(v)$ this gives

$$
\liminf _{n \rightarrow \infty}\left(-A v, J\left(x_{n}-v\right)\right) \leq 0 .
$$

Also we have, using $\left(\mathrm{J}_{4}\right)$ and the boundedness of $\left\{A x_{n}\right\}$, and $\left(\mathrm{J}_{2}\right)$

$$
\left(A x_{n}, J\left(x_{n}-v\right)\right)=\left(A x_{n}, J\left(x_{n}-v\right)-J\left(x_{n}-P_{n} v\right)\right)+\left(P_{n} A x_{n}, J\left(x_{n}-P_{n} v\right)\right) \longrightarrow 0 .
$$

It follows from (4.7), that

$$
\liminf \left(A x_{n}-A v, J\left(x_{n}-v\right)\right) \leq 0 .
$$

By the $s$-accretive property this gives $x_{k} \rightarrow v$ (a subsequence). Since $A$ is demicontinuous, it follows that $A x_{k} \rightarrow A v$. Now, for every $u \in X$,

$$
\left(A x_{k}, J\left(P_{k} u\right)\right)=\left(P_{k} A x_{k}, J\left(P_{k} u\right)\right) \longrightarrow 0 \quad \text { since } P_{k} A x_{k} \longrightarrow 0,
$$

and also $\left(A x_{k}, J\left(P_{k} u\right)\right) \rightarrow(A v, J(u))$ as $A x_{k} \rightarrow A v$ and $J$ is continuous. Since $J$ is surjective, $\left(\mathrm{J}_{3}\right)$, this proves that $A v=0$.

Remark 4.7. As a generalized contraction $A$ is continuous and bounded, Theorem 4.6 generalizes Theorem 2 in [22]. Note that in the above proof we do not need to suppose that the subspaces $X_{n}$ are nested, nor do we need to suppose that $P_{n}(K) \subset K$. However we often do need $P_{n}(K) \subset K$ in the fixed point theory of $A$-proper maps, see, for example [13], a condition that is usually met in applications, in which case $P_{n}(K)=$ $K_{n}=K \cap X_{n}$.

When $A$ is continuous and $P_{n}(K) \subset K$ it is possible to remove the boundedness assumption if we suppose that $A$ satisfies a slightly stronger condition: $A$ is said to be $S$-accretive if whenever $\left\{x_{n}\right\} \subset K$ is a bounded sequence and $y \in K$ and

$$
\liminf _{n \rightarrow \infty}\left(A x_{n}-A\left(P_{n} y\right), J\left(x_{n}-P_{n} y\right)\right) \leq 0
$$

then there is a subsequence $x_{n_{k}} \rightarrow y$.

Clearly this class includes strongly accretive continuous maps. It includes continuous $S$ - $\alpha$-accretive maps, that is, $\alpha$-accretive maps where $\alpha$ is lsc, that is, $y_{n} \rightarrow y$ and $r_{n} \rightarrow r$ imply $\alpha(y, r) \leq \liminf _{n \rightarrow \infty} \alpha\left(y_{n}, r_{n}\right)$. This holds, for example, if a map is $\alpha$-accretive and $\alpha(y, r)=\alpha(r)$. Similarly, we define the class of $S$ - $\psi$-dissipative maps.

TheOrem 4.8. Let $X$ be a reflexive $(U G) \pi_{1}$-space and let $K$ be a closed convex set with $P_{n}(K) \subset K$. Let $A: K \rightarrow X$ be a continuous, $S$-accretive map and suppose that $I-A$ is weakly inward relative to $K$. Then $A$ is A-proper at 0 . 
Proof. Let $x_{n} \in K_{n}$ be bounded and such that $P_{n} A x_{n} \rightarrow 0$. Since $A$ is continuous and $J$ is uniformly demicontinuous, we have

$$
\begin{aligned}
& \left(A\left(P_{n} v\right), J\left(x_{n}-P_{n} v\right)\right)-\left(A v, J\left(x_{n}-v\right)\right) \\
& \quad=\left(A\left(P_{n} v\right), J\left(x_{n}-P_{n} v\right)-J\left(x_{n}-v\right)\right)+\left(A\left(P_{n} v\right)-A v, J\left(x_{n}-v\right)\right) \longrightarrow 0
\end{aligned}
$$

so (4.7) gives $\liminf _{n \rightarrow \infty}\left(-A\left(P_{n} v\right), J\left(x_{n}-P_{n} v\right)\right) \leq 0$.

Using $\left(\mathrm{J}_{2}\right)$ we have $\left(A x_{n}, J\left(x_{n}-P_{n} v\right)\right)=\left(P_{n} A x_{n}, J\left(x_{n}-P_{n} v\right)\right) \rightarrow 0$ and so

$$
\liminf _{n \rightarrow \infty}\left(A x_{n}-A\left(P_{n} v\right), J\left(x_{n}-P_{n} v\right)\right) \leq 0 .
$$

By the $S$-accretive property there is a subsequence $x_{k} \rightarrow v$. By an argument similar to that at the end of the proof of Theorem 4.6, we have $A v=0$.

Corollary 4.9. Let $K$ be a closed convex subset of a $\pi_{1}$-space $X$ and let $T: K \rightarrow X$ be weakly inward relative to $K$. Suppose that either

(a) $X^{*}$ is uniformly convex and $T$ is a bounded, demicontinuous $\psi$-dissipative map, or

(b) $X$ is reflexive and $(U G)$ and $T$ is a continuous $S$ - $\psi$-dissipative map.

Then $I-T$ is A-proper at 0 . Also, for every $w \in K$ and $t \in[0,1], I-t T-(1-t) w$ is A-proper at 0 .

We say that a map $T: D \rightarrow X$ is of dissipative type if $T-c I$ is dissipative for some $c \in \mathbb{R}$, and, following Martin [15], we let $\mu[T]$ be the smallest such number $c$, so that

$$
(T x-T y, J(x-y)) \leq \mu[T]\|x-y\|^{2} .
$$

We consider dissipative type maps because these include contractive maps and enable us to give some fixed point theorems. We note that if $\mu[T]<1$, then $T$ is $\psi$-dissipative so we have the following corollary.

Corollary 4.10. If $T: K \rightarrow X$ is a weakly inward, dissipative type map with $\mu[T]<1$, and either

$\left(\mathrm{a}^{\prime}\right) X^{*}$ is uniformly convex and $T$ is bounded and demicontinuous, or

$\left(\mathrm{b}^{\prime}\right) X$ is a reflexive $(U G)$ space and $T$ is continuous.

Then $I-T$ is A-proper at 0 .

In the following, we state results when (a) of Corollary 4.9 holds (respectively, $\left(a^{\prime}\right)$ of Corollary 4.10), similar results are valid when (b) (respectively, $\left(b^{\prime}\right)$ ) holds. We leave the statement of these to the reader.

The above results prove $A$-properness at 0 , which is often adequate for proving fixed point theorems, but it is useful to have $A$-properness at more points. We now give such a result where $I+T$ (rather than $T$ ) is weakly inward. When $0 \in K$, as we have seen, this is stronger than assuming $T$ is weakly inward. If $K$ is a wedge, then $I+T$ weakly inward is equivalent to $T$ weakly inward. 
TheOREM 4.11. Let $K$ be a closed convex subset of a $\pi_{1}$-space $X$ with $X^{*}$ uniformly convex. Let $T: K \rightarrow X$ be a bounded, demicontinuous $\psi$-dissipative map and suppose that $I+T$ is weakly inward. Then $I-\lambda T$ is A-proper at all points of $K$ for every $\lambda \in[0,1]$.

Proof. Let $w \in K$ and let $B: K \rightarrow X$ be defined by $B x=w$. Then $B$ is trivially weakly inward and hence so is $B+\lambda T$ by Proposition 2.7. Also $B+\lambda T$ is $\psi_{\lambda}$-dissipative (with $\left.\psi_{\lambda}(y, r)=\lambda \psi(y, r)\right)$ so $I-(B+\lambda T)$ is $A$-proper at 0 , that is, $I-\lambda T$ is $A$-proper at $w$.

Note that this applies when $T$ is a generalized contraction.

Corollary 4.12. Let $X$ and $K$ be as in Theorem 4.11. Let $T: K \rightarrow X$ be a bounded, demicontinuous, dissipative type map and suppose that $I+T$ is weakly inward. Then $I-\lambda T$ is A-proper at all points of $K$ for every $\lambda \in[0, \delta]$ where $\delta$ is such that $\delta \mu[T]<1$.

Note that if $T$ is dissipative $(\mu[T] \leq 0)$, then this gives $I-\lambda T$ is $A$-proper at all points of $K$ for every $\lambda \geq 0$.

When maps are defined on the whole space, compact perturbations of $A$-proper maps are themselves $A$-proper. Recall that a map $C: D \rightarrow X$ is called compact if it is continuous and for every bounded subset $D_{0}$ of $D, \overline{C\left(D_{0}\right)}$ is a compact set. For maps defined only on a set $D$ we have the following result.

Theorem 4.13. Let $D, D_{1}$ be closed subsets of a $\pi_{c}$-space $X$ such that $D \cap D_{1} \neq$ $\emptyset$. Suppose that $A: D \rightarrow X$ is A-proper at all points of a closed set $E$ and that $C: D_{1} \rightarrow E$ is compact. Then $A-C: D \cap D_{1} \rightarrow X$ is A-proper at 0 .

Proof. Suppose that $P_{n}(A-C)\left(x_{n}\right) \rightarrow 0$ for a bounded sequence $\left\{x_{n}\right\}$ with $x_{n} \in$ $D \cap D_{1} \cap X_{n}$. Then, by compactness of $C$, there is a subsequence $\left\{x_{k}\right\}$ such that $C x_{k} \rightarrow w \in E$. Then $P_{k} A x_{k} \rightarrow w$ and as $A$ is $A$-proper on $E$ we obtain (a further subsequence) $x_{k} \rightarrow x \in D \cap D_{1}$ with $A x=w$. As $C$ is continuous we have $w=C x$ and so $A x-C x=0$.

Corollary 4.14. Let $X$ and $K$ be as in Theorem 4.11. Let $T: K \rightarrow X$ be a bounded, demicontinuous $\psi$-dissipative map with $I+T$ weakly inward. Let $D$ be a closed subset of $K$ and $C: D \rightarrow K$ be compact. Then $I-\lambda T-C: D \rightarrow X$ is A-proper at 0 for every $\lambda \in[0,1]$.

\section{Fixed point theorems}

Let $K$ be a closed convex set in a $\pi_{1}$-space $X$. We shall from now on assume that $P_{n} K \subset K$ so that $P_{n}(K)=K \cap X_{n}$. This mild restriction ensures that if $T: K \rightarrow X$ is weakly inward relative to $K$, then $P_{n} T: K_{n} \rightarrow X_{n}$ is weakly inward relative to $K_{n}$ and seems to be a necessary requirement in the theory of the fixed point index for weakly inward $A$-proper maps (see [13]). 
Let $G$ be an open set in $X$. We denote by $\bar{G}_{K}$ and $\partial_{K} G$ the closure and the boundary, respectively, of $G_{K}=G \cap K$ relative to $K$.

We first recall the definition of fixed point index for a continuous weakly inward map in a finite-dimensional Banach space from [13]. Certain retractions are used.

Definition 5.1. An $\varepsilon$-retraction of $X$ onto $K$ is a continuous map $r: X \rightarrow K$ which satisfies

$$
\|x-r(x)\| \leq(1+\varepsilon) \operatorname{dist}(x, K), \quad \text { for every } x \in X .
$$

By the Dugundji extension theorem (cf. [9, Section 18]), $\varepsilon$-retractions exist for every $\varepsilon>0$. In fact, in finite-dimensional spaces nearest points always exist and are unique if the space is strictly convex. Hence a 0 -retraction (usually termed metric projection) exists when the space is strictly convex. It is possible to change to an equivalent strictly convex norm which is arbitrarily close to the original norm. (For the infinite-dimensional result in a reflexive space, see [3].) This gives an alternative way of obtaining an $\varepsilon$ retraction.

Definition 5.2. Let $K$ be a closed convex set in a finite-dimensional Banach space $X$ and let $G$ be a bounded open subset of $X$ such that $G_{K} \neq \emptyset$. Let $f: \bar{G}_{K} \rightarrow X$ be continuous and weakly inward relative to $K$. Suppose also that $x \neq f(x)$ for all $x \in \partial_{K} G$. Define the fixed point index by the equation

$$
i_{K}\left(f, G_{K}\right)=i_{K}\left(r_{\varepsilon} f, G_{K}\right),
$$

for $\varepsilon$ sufficiently small, where $r_{\varepsilon}: X \rightarrow K$ is an $\varepsilon$-retraction and $i_{K}\left(r_{\varepsilon} f, G_{K}\right)$ is the classical fixed point index as defined, for example, in Amann [1].

In [13] it is shown that the fixed point index defined above is independent of the particular $\varepsilon$-retraction provided $\varepsilon$ is sufficiently small and has the usual properties. We state those which we use.

$\left(\mathrm{P}_{1}\right)$ (Existence) If $i_{K}\left(f, G_{K}\right) \neq 0$, then $f$ has a fixed point in $G_{K}$.

$\left(\mathrm{P}_{2}\right)$ (Normalisation) If $u \in G_{K}$, then $i_{K}\left(\hat{u}, G_{K}\right)=1$, where $\hat{u}(x)=u$ for $x \in \bar{G}_{K}$.

$\left(\mathrm{P}_{3}\right)$ (Homotopy property) Let $h:[0,1] \times \bar{G}_{K} \rightarrow X$ be continuous and such that $h(t, \cdot): \bar{G}_{K} \rightarrow X$ is weakly inward for each $t \in[0,1]$. If $x \neq h(t, x)$ for $x \in$ $\partial G_{K}$ and $t \in[0,1]$, then

$$
i_{K}\left(h(0, \cdot), G_{K}\right)=i_{K}\left(h(1, \cdot), G_{K}\right) .
$$

We now prove a new fixed point result which is sufficiently general to cover our needs. Note that we do not assume that $T$ is bounded. Also, we do not need $I-t T-$ $(1-t) w$ to be $A$-proper at 0 for every $t \in[0,1]$, and some $w \in K$.

Theorem 5.3. Let $K$ be a closed, convex set in $X$ and suppose $T: K \rightarrow X$ is weakly inward relative to $K$ and $I-T$ is A-proper at 0 . If $K$ is unbounded suppose also there exist $w \in K, R>\|w\|$, and $N \in \mathbb{N}$ such that

$$
x \neq t P_{n} T x+(1-t) P_{n} w \quad \text { for } x \in K,\|x\|=R, 0 \leq t \leq 1, n \geq N .
$$


Then $T$ has a fixed point in $K$.

Proof. Since $T: K \rightarrow X$ is weakly inward relative to $K$, and $P_{n}(K) \subset K$,

$$
t P_{n} T+(1-t) P_{n} \hat{w}: K_{n} \longrightarrow X_{n}
$$

is weakly inward relative to $K_{n}$ for every $t \in[0,1]$. By the homotopy property of the finite-dimensional index we have for $n \geq N$

$$
i_{K_{n}}\left(P_{n} T, B_{K_{n}}(R)\right)=i_{K_{n}}\left(P_{n} \hat{w}, B_{K_{n}}(R)\right)=1 .
$$

Thus $P_{n} T$ has a fixed point in $K_{n} \cap B_{R}$, that is, there exists $x_{n}$ such that $x_{n}-P_{n} T x_{n}=0$. As $I-T$ is $A$-proper at 0 this gives a fixed point of $T$ in $K \cap \bar{B}_{R}$.

Remark 5.4. The relation (5.4) is an a priori bound requirement. Clearly (5.4) is satisfied if $T(K)$ is a bounded set. When $K$ is bounded the conclusion of the theorem can be deduced from the finite-dimensional version of a fixed point theorem of Halpern and Bergman [7] without recourse to the fixed point index theory. When $T(K)$ is bounded, the conclusion of Theorem 5.3 has been obtained from the index theory in [13].

We give some new fixed point theorems. It is convenient to prove an a priori bound result first.

Proposition 5.5. Let $K$ be a closed, unbounded set and let $T: K \rightarrow X$ be a demicontinuous $\psi$-dissipative map. Let $C: K \rightarrow X$ be a map such that there exist constants $M_{0}, 0 \leq \gamma \leq 1$ such that $\limsup _{\|x\| \rightarrow \infty}\|C x\| /\|x\|^{\gamma} \leq M_{0}, x \in K$. Suppose that there exist $w \in K$ and $N \in \mathbb{N}$ such that, for every $n \geq N, \liminf _{r \rightarrow \infty}\left(r^{2}-\right.$ $\left.\psi\left(P_{n} w, r\right)\right) / r^{1+\gamma} \geq M>M_{0}$ when $\gamma>0$ and $\lim _{r \rightarrow \infty}\left(r^{2}-\psi\left(P_{n} w, r\right)\right) / r=\infty$ when $\gamma=0$. Then there exists $R$ such that

$$
x \neq t P_{n} T x+t P_{n} C x+(1-t) P_{n} w \quad \text { for } x \in K_{n},\|x\|=R, 0 \leq t \leq 1, n \geq N .
$$

Proof. Let $\varepsilon>0$ be such that $M_{0}+2 \varepsilon<M$. For $\gamma>0$, using $\left\|P_{n} w\right\| \leq\|w\|$ and the inequality $(1+s)^{\gamma} \leq 1+\left(2^{\gamma}-1\right) s$ for $0 \leq s \leq 1$, we see that there is $R_{1}$ such that $\|C x\| \leq\left(M_{0}+\varepsilon\right)\left\|x-P_{n} w\right\|^{\gamma}$ for $\|x\| \geq R_{1}$, for all $n$. Suppose that

$$
x=t P_{n} T x+t P_{n} C x+(1-t) P_{n} w \quad \text { for some } t \in[0,1], x \in K_{n},\|x\| \geq R_{1} .
$$

Then

$$
\begin{aligned}
\left\|x-P_{n} w\right\|^{2} & =\left(t P_{n} T x+t P_{n} C x-t P_{n} w, J\left(x-P_{n} w\right)\right) \\
& =t\left(T x-T P_{n} w, J\left(x-P_{n} w\right)\right)+t\left(T P_{n} w-w, J\left(x-P_{n} w\right)\right)+t\left(C x, J\left(x-P_{n} w\right)\right) \\
& \leq \psi\left(P_{n} w,\left\|x-P_{n} w\right\|\right)+\left(\left\|T P_{n} w\right\|+\|w\|\right)\left\|x-P_{n} w\right\|+\|C x\|\left\|x-P_{n} w\right\| .
\end{aligned}
$$

Hence we obtain

$$
\frac{\left\|x-P_{n} w\right\|^{2}-\psi\left(P_{n} w,\left\|x-P_{n} w\right\|\right)}{\left\|x-P_{n} w\right\|^{1+\gamma}} \leq \frac{\left(\left\|T P_{n} w\right\|+\|w\|\right)}{\left\|x-P_{n} w\right\|^{\gamma}}+M_{0}+\varepsilon .
$$


Since $P_{n} w \rightarrow w$ and $T$ is demicontinuous, $\left\|T\left(P_{n} w\right)\right\|$ are uniformly bounded, say, $\left\|T P_{n} w\right\| \leq M_{2}$ for all $n$. Therefore, there exists $R_{2} \geq R_{1}$ such that $\left\|x-P_{n} w\right\| \geq R_{2}$ implies $\left(\left\|T P_{n} w\right\|+\|w\|\right) /\left\|x-P_{n} w\right\|^{\gamma}<\varepsilon$. This would contradict the hypothesis so we have $\|x\| \leq R_{2}+\|w\|$. There is an obvious modification if $\gamma=0$.

We first consider the case when $C \equiv 0$. $T$ is taken to be weakly inward and we obtain a unique fixed point.

Theorem 5.6. Let $X$ be a $\pi_{1}$-space with $X^{*}$ uniformly convex and let $K$ be a closed convex set. Let $T: K \rightarrow X$ be bounded, demicontinuous weakly inward $\psi$-dissipative map. If $K$ is unbounded, assume also that there exist $w \in K$ and $N \in \mathbb{N}$ such that

$$
\lim _{r \rightarrow \infty} \frac{r^{2}-\psi\left(P_{n} w, r\right)}{r}=\infty, \quad \forall n \geq N
$$

Then $T$ has a unique fixed point in $K$.

Proof. By Corollary 4.9, $I-T$ is $A$-proper at 0 . Theorem 5.3 applies using the a priori bound argument from Proposition 5.5 with $\gamma=0$ when $K$ is unbounded. We obtain uniqueness as follows. Since

$$
(T x-T y, J(x-y)) \leq \psi(y,\|x-y\|),
$$

if $T x=x$ and $T y=y$, then $\|x-y\|^{2} \leq \psi(y,\|x-y\|)<\|x-y\|^{2}$ unless $x=y$.

Note that Theorem 5.6 applies in particular to a dissipative type map $T$ with $\mu[T]<$ 1. The result also holds when $T$ is a continuous $S$ - $\psi$-dissipative map and $X$ is (UG).

Corollary 5.7. Let $X$ and $K$ be as in Theorem 5.6 and let $A: K \rightarrow X$ be a bounded, demicontinuous mapping with $I-A$ weakly inward and satisfying $(A x-A y, J(x-y))$ $\geq \alpha(\|x-y\|)\|x-y\|$, where $\alpha(r)>0$ for $r>0$. If $K$ is unbounded, assume also that $\lim _{r \rightarrow \infty} \alpha(r)=\infty$. Then there is a unique $x \in K$ such that $A x=0$.

Proof. $T=I-A$ is $\psi$-dissipative with $\psi(r)=r^{2}-\alpha(r) r$.

Remark 5.8. Theorem 5.6 is an extension of Theorem 7.3 [15, page 257], which deals with continuous maps $T$ satisfying $\mu[T]<1$ and the tangency condition (1.2) rather than the geometrical condition $I+T$ weakly inward. However, Martin's result applies in arbitrary Banach spaces and to arbitrary closed sets $K$, and is proved by entirely different methods. Corollary 5.7 is stated so as to compare with Theorem 1 of [5]. It is an extension in some respects: in [5] stronger assumptions are made on $\alpha, A$ is continuous, but $X$ is arbitrary and $K$ is an arbitrary closed set. Obviously our Theorem 5.6 allows a slightly more general result than stated in Corollary 5.7.

Theorem 5.9. Let $X$ be a $\pi_{1}$-space with $X^{*}$ uniformly convex and $K$ a (bounded or unbounded) closed convex set. Let $T: K \rightarrow X$ be a bounded demicontinuous dissipative type map with $I+T$ weakly inward. Let $\delta \geq 0$ be such that $\delta \mu[T]<1$. Then $(I-\lambda T)(K) \supseteq K$ for every $\lambda \in[0, \delta]$. 
Proof. For an arbitrary $w \in K, \lambda T+w$ is weakly inward (as in Theorem 4.11) and $\mu[\lambda T+w]=\lambda \mu[T]<1$. Therefore, $\lambda T+w$ has a unique fixed point $x$ and $x-$ $\lambda T x=w$.

Remark 5.10. Theorem 5.9 has a partial converse. If $T$ is continuous, then $(I-\lambda T)(K)$ $\supseteq K$ for all small $\lambda>0$ implies that $I+T$ is weakly inward in an arbitrary Banach space. If $X$ is uniformly convex this holds without assuming continuity of $T$. This can be deduced from results of Crandall, see [23] and the references therein. Theorem 5.9 is given by Martin [15] when $T$ is continuous in an arbitrary Banach space, by very different methods.

We now consider fixed point theorems for sums of dissipative type and compact maps.

Theorem 5.11. Let $X$ be a $\pi_{1}$-space with $X^{*}$ uniformly convex and $K$ a (bounded or unbounded) closed convex set. Let $C: K \rightarrow K$ be compact and let $T: K \rightarrow X$ be a bounded demicontinuous $\psi$-dissipative map with $I+T$ weakly inward relative to $K$. If $K$ is unbounded, assume also that there exist $\gamma \in[0,1]$ and $M>0$ such that $\limsup _{\|x\| \rightarrow \infty, x \in K}\|C x\| /\|x\|^{\gamma} \leq M$. Suppose also that there exist $w \in K$ and $N \in \mathbb{N}$ such that, for every $n \geq N, \liminf _{r \rightarrow \infty}\left(r^{2}-\psi\left(P_{n} w, r\right)\right) / r^{1+\gamma} \geq M_{1}>M$ when $\gamma>0$ and $\lim _{r \rightarrow \infty}\left(r^{2}-\psi\left(P_{n} w, r\right)\right) / r=\infty$ when $\gamma=0$. Then $T+C$ has a fixed point in $K$.

Proof. By Corollary 4.14, $I-T-C$ is $A$-proper at 0 . Theorem 5.3 applies using the a priori bound argument from Proposition 5.5.

Note that if $\mu[T]<1$ then $r^{2}-\psi\left(P_{n} w, r\right)=(1-\mu[T]) r^{2}$ so Theorem 5.11 applies if $C$ satisfies $\|C x\| /\|x\| \rightarrow 0$ as $\|x\| \rightarrow \infty$.

\section{Application}

As an application of Theorem 5.11 we consider the problem of finding positive solutions of the integral equation

$$
u(x)=\lambda g(x, u(x))+\int_{\Omega} h(x, y) f(y, u(y)) d y,
$$

where $\Omega$ is a bounded domain in $\mathbb{R}^{n}$.

Such equations were considered in $L^{p}(\Omega)$ in [10], where the fixed point index for $A$-proper self-maps was applied and the function $g$ was required to be positive. Existence of positive solutions was considered in the Hilbert space $L^{2}(\Omega)$ in [11], where variational methods were used but the function $g$ was allowed to have some negative values.

In this section, we consider existence of positive solutions of (6.1) by using Theorem 5.11 and we allow $g$ to have some negative values.

Equation (6.1) can be written as $u=\lambda T u+C u$ in a suitable function space. Here we consider the Banach space $X=L^{p}(\Omega)$, where $1<p<\infty$ and $\Omega$ is a bounded domain in $\mathbb{R}^{n}$. The norm in $L^{p}$ is denoted $\|\cdot\|_{p}$. The dual space is $L^{p^{\prime}}(\Omega)$, where $1 / p+1 / p^{\prime}=1$, 
which is uniformly convex. We seek solutions in the cone $K=L_{+}^{p}=\left\{u \in L^{p}(\Omega)\right.$ : $u(x) \geq 0$ a.e. on $\Omega\}$. It is known that $L^{p}$ is a $\pi_{1}$-space and that $P_{n}(K) \subset K$.

THEOREM 6.1. Assume that the following conditions hold:

$\left(\mathrm{H}_{1}\right) g: \Omega \times \mathbb{R}^{+} \rightarrow \mathbb{R}$ satisfies Carathéodory conditions and

(i) $g(x, 0) \geq 0$ for all $x \in \Omega$ and $g(x, 0) \in L^{p}(\Omega)$;

(ii) there exists $k \in[0,1)$ such that for every $x \in \Omega$ and all $u, v \geq 0$

$$
(g(x, u)-g(x, v))(u-v) \leq k|u-v|^{2} ;
$$

(iii) there exist $m \geq 0$ and $a \in L^{p}(\Omega)$ such that for all $x \in \Omega$ and $u \geq 0$,

$$
g(x, u) \geq-m u-a(t) .
$$

$\left(\mathrm{H}_{2}\right) f: \Omega \times \mathbb{R}^{+} \rightarrow \mathbb{R}^{+}$satisfies Carathéodory conditions and there exist $q \in$ $(p, \infty), c>0$ and a positive function $b \in L^{q}(\Omega)$ such that

$$
f(x, u) \leq b(x)+c u^{p / q} \quad \forall x \in \Omega \text { and } u \geq 0 .
$$

$\left(\mathrm{H}_{3}\right) h: \Omega \times \Omega \rightarrow \mathbb{R}^{+}$is a bounded, measurable function with $\{h(\cdot, y): y \in \Omega\}$ equicontinuous.

Then, for each $\lambda \in[0,1]$, equation (6.1) has at least one solution in $K$.

Proof. Without loss of generality, we may, and do, take $\lambda=1$. Define a map $F: K \subset$ $L^{p} \rightarrow L_{+}^{q}$ by $(F u)(x)=f(x, u(x))$. Then condition $\left(\mathrm{H}_{2}\right)$ implies that $F: K \rightarrow L_{+}^{q}$ is continuous and bounded. Also, the linear map $L: L^{q}(\Omega) \rightarrow L^{p}(\Omega)$ defined by

$$
L v(x)=\int_{\Omega} h(x, y) v(y) d y
$$

is compact, by Proposition 4.3 [15, page 176]. Hence, $C: \equiv L F: K \rightarrow K$ is compact.

By conditions (ii) and (iii) we have

$$
|g(x, u)| \leq \max \{k, m\} u+\max \{g(x, 0), a(x)\} \quad \forall x \in \Omega \text { and } u \geq 0 .
$$

Therefore, $T: K \rightarrow X$ defined by $T u(x)=g(x, u(x))$ is a bounded continuous map. The duality map $J: L^{p} \rightarrow L^{p^{\prime}}$ is given by

$$
J(u)(x)=\|u\|_{p}^{2-p}|u(x)|^{p-2} u(x)
$$

and so $T$ satisfies

$$
(T u-T v, J(u-v)) \leq k\|u-v\|_{p}^{2} \quad \forall u, v \in K .
$$

Thus, $T: K \rightarrow L^{p}(\Omega)$ is of dissipative type with $\mu[T] \leq k<1$.

We show that $T$ is weakly inward by using Lemma 2.3. Let $u \in K$ be such that $\left(u, u^{*}\right)=0$ for some $u^{*} \in K^{*}$. Then $u^{*}$ can be identified with an $L^{p^{\prime}}$ function, $u^{*}(x) \geq 0$ 
a.e. and $\int_{\Omega} u(x) u^{*}(x) d x=0$, so that $u^{*}(x)=0$ a.e. on the set $\{x \in \Omega: u(x)=0\}$. Therefore, we have

$$
\left(T u, u^{*}\right)=\int_{\Omega} g(x, u(x)) u^{*}(x) d x=\int_{\{x \in \Omega: u(x)=0\}} g(x, 0) u^{*}(x) d x \geq 0 .
$$

It follows from Lemma 2.3 that $T$ is weakly inward relative to $K$, hence $I+T$ is weakly inward since $K$ is a cone.

We show that $\lim _{\|u\|_{p} \rightarrow \infty}\|C u\|_{p} /\|u\|_{p}=0$. Let $M=\sup \{h(x, y): x, y \in \Omega\}$. Then $M<\infty$ by assumption. Using $\left(\mathrm{H}_{2}\right)$ we have

$$
\begin{aligned}
\|C u\|_{p} & =\left[\int_{\Omega}\left|\int_{\Omega} h(x, y) f(y, u(y)) d y\right|^{p} d x\right]^{1 / p} \\
& \leq M(\operatorname{meas}(\Omega))^{1 / p}\left[\int_{\Omega}\left(b(x)+c(u(x))^{p / q}\right) d x\right]^{1 / p} \\
& \leq M(\operatorname{meas}(\Omega))^{1 / p}\left[\int_{\Omega} b(x) d x+c(\operatorname{meas}(\Omega))^{1 / p}\left(\int_{\Omega}(u(x))^{p} d x\right)^{1 / q}\right]^{1 / p} .
\end{aligned}
$$

Since $q>p, \lim _{\|u\|_{p} \rightarrow \infty}\|C u\|_{p} /\|u\|_{p}=0$. The result now follows immediately from Theorem 5.11.

\section{References}

[1] H. Amann, Fixed point equations and nonlinear eigenvalue problems in ordered Banach spaces, SIAM Rev. 18 (1976), no. 4, 620-709. MR 54\#3519. Zbl 345.47044.

[2] T. D. Benavides, Some properties of the set and ball measures of noncompactness and applications, J. London Math. Soc. (2) 34 (1986), no. 1, 120-128. MR 87k:47124. Zbl 593.47051.

[3] H. Brezis, M. G. Crandall, and A. Pazy, Perturbations of nonlinear maximal monotone sets in Banach space, Comm. Pure Appl. Math. 23 (1970), 123-144. MR 41\#2454. Zbl 182.47501.

[4] F. E. Browder and D. G. de Figueiredo, J-monotone nonlinear operators in Banach spaces, Nederl. Akad. Wetensch. Proc. Ser. A 6928 (1966), 412-420. MR 34\#4957. Zbl 148.13602.

[5] K. Deimling, Zeros of accretive operators, Manuscripta Math. 13 (1974), 365-374. MR 50\#3030. Zbl 288.47047.

[6] _ Nonlinear Functional Analysis, Springer-Verlag, Berlin, New York, 1985. MR 86j:47001. Zbl 559.47040.

[7] B. R. Halpern and G. M. Bergman, A fixed-point theorem for inward and outward maps, Trans. Amer. Math. Soc. 130 (1968), 353-358. MR 36\#4397. Zbl 153.45602.

[8] W. A. Kirk, On nonlinear mappings of strongly semicontractive type, J. Math. Anal. Appl. 27 (1969), 409-412. MR 39\#6128. Zbl 183.15103.

[9] M. A. Krasnosel'skiı̌ and P. P. Zabrě̌ko, Geometrical Methods of Nonlinear Analysis, Grundlehren der Mathematischen Wissenschaften [Fundamental Principles of Mathematical Sciences], vol. 263, Springer-Verlag, Berlin, New York, 1984, Translated from the Russian by Christian C. Fenske. MR 85b:47057. Zbl 546.47030. 
$100 A$-properness and fixed point theorems for dissipative type maps

[10] B. Lafferriere and W. V. Petryshyn, New positive fixed point and eigenvalue results for $P_{\gamma}$ compact maps and some applications, Nonlinear Anal. 13 (1989), no. 12, 1427-1440. MR 90k:47115. Zbl 702.47036.

[11] K. Lan and J. Webb, Variational inequalities and fixed point theorems for PM-maps, J. Math. Anal. Appl. 224 (1998), no. 1, 102-116. MR 99g:47149. Zbl 942.49011.

[12] K. Q. Lan and J. R. L. Webb, A-properness of contractive and condensing maps, to appear in Nonlinear Anal.

[13] - A fixed point index for weakly inward A-proper maps, Nonlinear Anal. 28 (1997), no. 2, 315-325. MR 97g:47063. Zbl 883.47074.

[14] J. T. Marti, Introduction to the Theory of Bases, Springer Tracts in Natural Philosophy, vol. 18, Springer-Verlag New York Inc., New York, 1969. MR 55\#10994. Zbl 191.41301.

[15] R. H. Martin, Jr., Nonlinear Operators and Differential Equations in Banach Spaces, Pure and Applied Mathematics, Wiley-Interscience [John Wiley \& Sons], New York, London, Sydney, 1976. MR 58\#11753. Zbl 333.47023.

[16] W. V. Petryshyn, Nonlinear equations involving noncompact operators, Nonlinear Functional Analysis (Proc. Sympos. Pure Math. Part 1, Chicago, Ill., 1968), vol. 18, Amer. Math. Soc., Providence, R.I., 1970, pp. 206-233. MR 42\#6670. Zbl 232.47070.

[17] _ Approximation-Solvability of Nonlinear Functional and Differential Equations, Monographs and Textbooks in Pure and Applied Mathematics, vol. 171, Marcel Dekker Inc., New York, 1993. MR 94f:47081. Zbl 772.65040.

[18] _ Generalized Topological Degree and Semilinear Equations, Cambridge Tracts in Mathematics, vol. 117, Cambridge University Press, Cambridge, 1995. MR 96k:47103. Zbl 834.47053.

[19] S. Reich, Product formulas, nonlinear semigroups, and accretive operators, J. Funct. Anal. 36 (1980), no. 2, 147-168. MR 81k:47076. Zbl 437.47048.

[20] J. R. L. Webb, Remarks on k-set contractions, Boll. Un. Mat. Ital. (4) 4 (1971), 614-629. MR 45\#2544. Zbl 219.47058.

[21] On a property of duality mappings and the A-properness of accretive operators, Bull. London Math. Soc. 13 (1981), no. 3, 235-238. MR 82f:47065. Zbl 476.47039.

[22] _ A-properness and fixed points of weakly inward mappings, J. London Math. Soc. (2) 27 (1983), no. 1, 141-149. MR 85b:47065. Zbl 508.47058.

[23] _ Zeros of weakly inward accretive mappings via A-proper maps, Theory and Applications of Nonlinear Operators of Accretive and Monotone Type, Lecture Notes in Pure and Appl. Math., vol. 178, Dekker, New York, 1996, pp. 289-297. MR 97e:47091. Zbl 883.47065.

K. Q. Lan: Department of Mathematics and Statistics, York University, 4700 Keele Street, Toronto, Ontario, Canada M3J 1P3

E-mail address: kunquan@yorku.ca

J. R. L. Webb: Department of Mathematics, University of Glasgow, Glasgow G12 8QW, UK

E-mail address: jrlw@maths.gla.ac.uk 


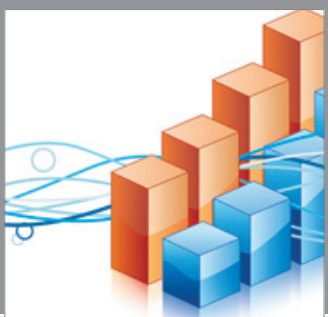

Advances in

Operations Research

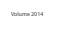



\section{The Scientific} World Journal
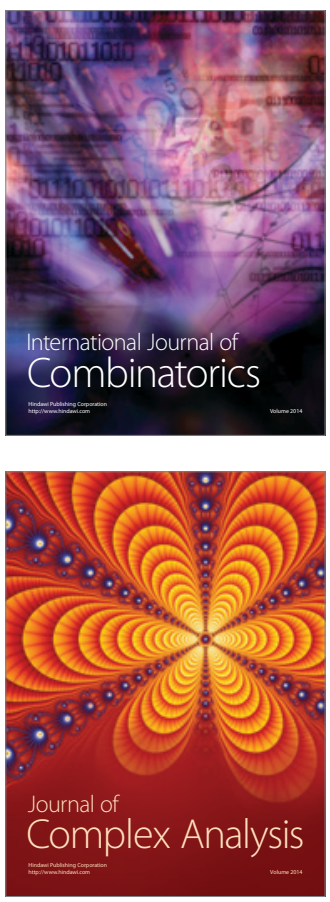

International Journal of

Mathematics and

Mathematical

Sciences
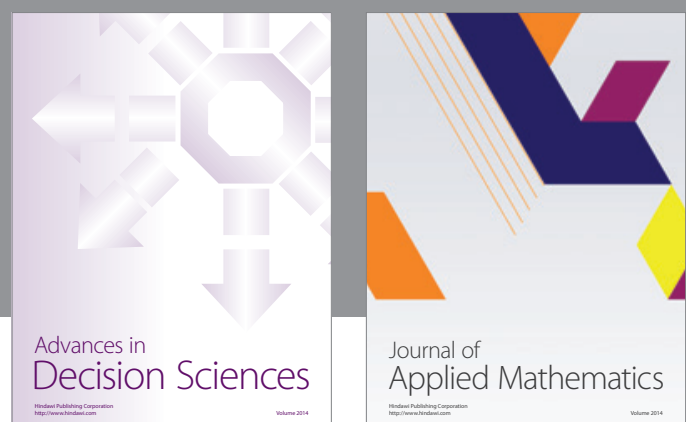

Journal of

Applied Mathematics
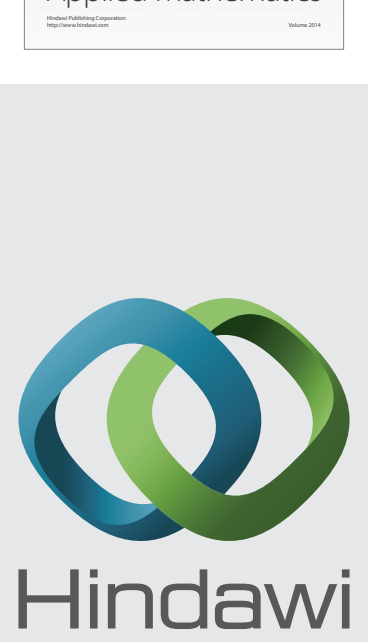

Submit your manuscripts at http://www.hindawi.com
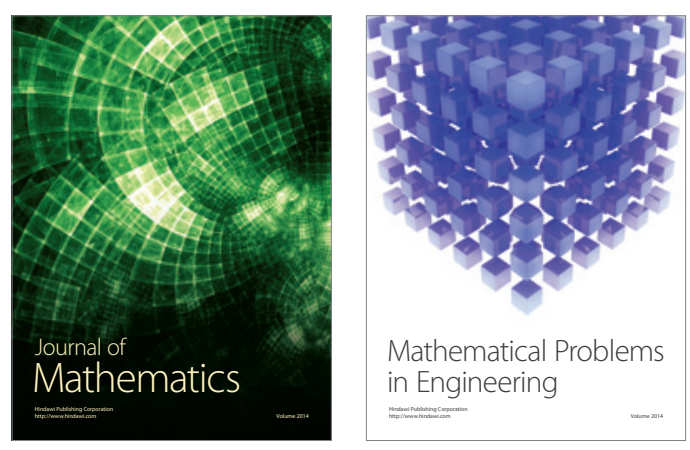

Mathematical Problems in Engineering
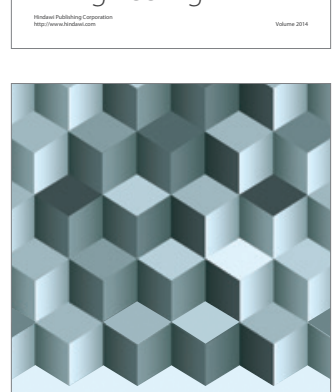

Journal of

Function Spaces
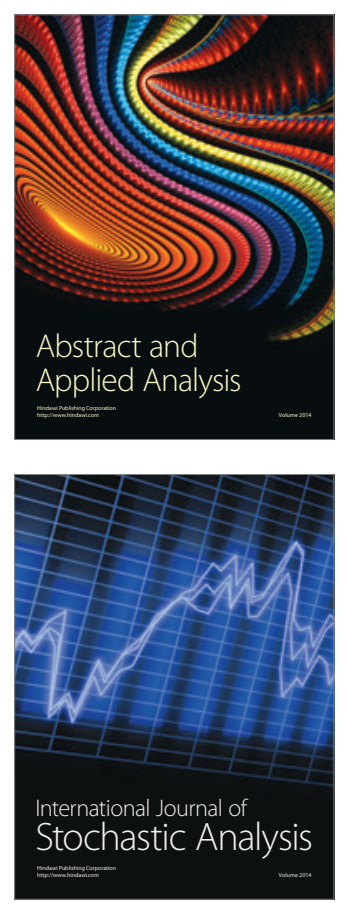



ournal of

Probability and Statistics

Promensencen
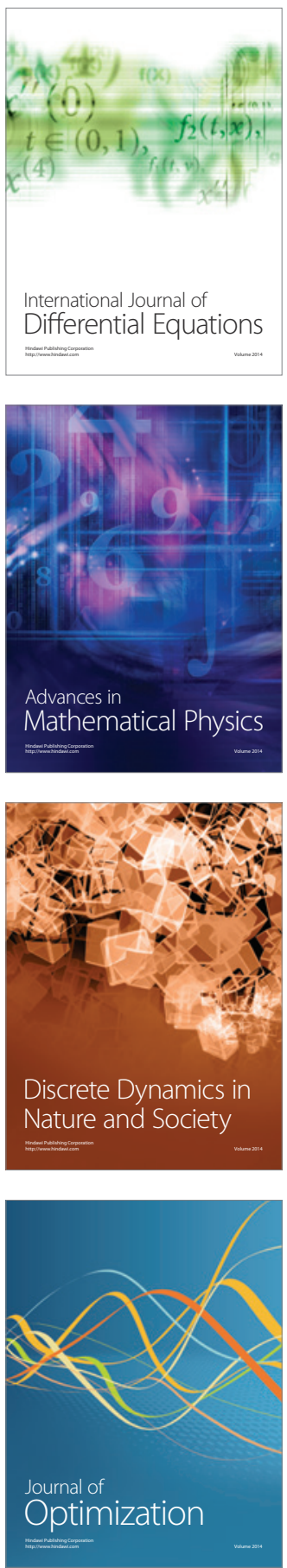